\title{
TIME SERIES ANALYSIS: FORECASTING SALES PERIODS IN WHOLESALE SYSTEMS
}

\author{
$U D C((544.42 / .43: 004.738 .5): 658.7)$
}

\author{
Bratislav Predić, Nevena Radosavljević, Aleksandar Stojčić \\ University of Niš, Faculty of Electronic Engineering, Republic of Serbia
}

\begin{abstract}
The main goal of time series analysis is explaining the correlation and the main features of the data in chronological order by using appropriate statistical models. It is being used in various aspects of life and work, as well as in forecasting future product demands, service demands, etc. The most common type of time series data is the one whose observations are taken in equally distributed time intervals (daily, weekly, monthly, etc.). However, in this paper, we analyze a different kind of time series which represents product purchase moments. Thus, since there are not any regular observation periods, this irregular time series must be transformed in some way before traditional methods of analysis can be applied. After the data transformation is complete, the next step is modeling the nonstationary time series using commonly known models such as ARIMA and PNBD, which have been chosen for their fairly easy and successful forecasting processes. The goal of this analysis is timely product advertising to a customer in order to increase sales.

Unlike some other models that consider the relationship between two or more different phenomena, time series models, including ARIMA, Pareto/NBD and Poisson models, examine the impact of historical values of a single phenomenon on its present and future value. This approach enables the study of the behavior of a given phenomenon over time and produces good results, especially if a large amount of historical data is available.
\end{abstract}

Key words: time series analysis, product demands, seasonality, ARIMA, PNBD, Poisson, forecasting

Received September 17, 2019

Corresponding author: Bratislav Predić

Faculty of Electronic Engineering, Aleksandra Medvedeva 14, 18000 Niš, Republic of Serbia

E-mail: bratislav.predic@elfak.ni.ac.rs 


\section{INTRODUCTION}

Time series are a common part of everyday life and work. Thus, their analysis is a statistical discipline which has seen the most dynamic development in the past decade. Considering the fact that the decision-making process is often closely related to forecasting future values of time dependent variables, the time series analysis plays a very important role in this process. In this context, forecasting future values represents an analysis of historical data and using a corresponding mathematical method to extrapolate future revenue.

The main subject of this paper is irregular time series which hold each purchase timestamp and quantity the customer has bought. The main cause of irregularity in this time series is the fact that time intervals between samples are unequal to one another; samples are not being taken in regular time intervals (daily, weekly, etc.,), but instead at the precise moment of the purchase. For this reason, time series like this one cannot be analyzed and modeled by standard methods. Certain mathematical transformations have to be performed on the data, before continuing with the regular time series analysis. The general idea is to calculate the average daily consumption of some product for the customer, based on the time period between two purchases and quantity of the product which a customer has bought. In this way, we get regular time series of average consumption on a daily level.

ARMA (Autoregressive Moving Model) model belongs to a group of stochastic models which may be used in a simulation as well as in a time series analysis. If the time series is nonstationary, as in this case, but can be reduced to stationary, the ARIMA (Autoregressive Integrated Moving Model) model is the appropriate model for analysis. The seasonal ARIMA model comes from the standard ARIMA model, but as an important factor includes seasonal component of the time series. As a matter of fact, the seasonal ARIMA model is one of the most powerful models for time series analysis considering the fact it can fit most time series data, regardless of their seasonal component's regularity. Box and Jenkins were pioneers of this methodology in the seventies.

Considering the complexity of mathematical calculations in the model building process, this paper will rely mostly on the existing statistical software solutions- programming language $\mathrm{R}$, for calculations and creating related graphics.

\section{PROBLEM DOMAIN AND RELATED WORK}

When it comes to purchase analysis and processing those kinds of data, most papers, as well as commercial systems, are focusing on so called, recommender systems. The main characteristics of these systems is the fact that based on series of previous purchases, personalized recommendations are generated for existing customers. The domain of these solutions is closely related to transactions between business and consumers (B2C). The other type of transactions, business to business, or shorter, B2B, is the one rarely seen in papers. Some of the main differences [1] between these two types of transactions are shown in Error! Reference source not found. 
Table 1 Comparison of transaction types

\begin{tabular}{ll}
\hline Wholesale & Retail \\
\hline Purchase in bulk. & Purchase of few items. \\
Decisions about purchase are being made by board. Decisions are made by an individual. \\
Longer time periods between purchases. & Very short time periods between \\
& purchases. \\
Rational decision. & Emotional decisions. \\
Purchase at need. & Desire driven purchases. \\
\hline
\end{tabular}

All of this points the fact that the B2B type of purchase is a more stable process in general. Rationality of decisions, longer time period between purchases, as well as larger bought quantity are characteristics of a process driven by some rules, and as such less prone to random events.

Most of the research on this topic comes from the field of marketing. Papers dealing with this topic, in addition to the time of next purchase, usually predict future values of other variables, such as brand choice and quantity.

Already existing solutions in this area can be divided into two larger units. The first group of solutions starts with the assumption that behind each purchase there is a motive, that is, the decision to make is: 'whether to buy?'. The second approach does not question the circumstances under which the transaction occurred but looks at them from the perspective of the time distance between every two purchases. This approach aims to find the answer to the question 'when to buy?'.

\subsection{Whether to buy?}

As already pointed out, this group is characterized by a buying motive. The basic idea behind this approach is that the consumer purchases only when the need arises. The models from this group perform better when applied to the data from a B2B business. The reason for this is rational, need-driven purchasing, which are the hallmarks of this type of business. One of the representatives of this group is addressed in [2]. The authors of this paper assume that every purchase is made when inventory falls below a certain limit. Modeling is done separately for each segment (product). Each edited buyer-product pair is assigned two variables:

$\mathbf{C R}^{\mathbf{h}}$ - spending rate for the customer $h$

$\mathbf{I N V}_{\mathbf{t}}^{\mathbf{h}}$ - inventory at the time $t$ for the customer $h$

Both variables affect the probability of buying (with some coefficient). Consumption rates have a positive impact on the likelihood of a purchase since the idea is that higherend customers have more reason to make a purchase. The rate of consumption for a customer is calculated in the initialization period, namely: by finding the average value of weekly consumption during this period. In contrast to the consumption rate which has a positive correlation to the purchase probability, the inventory has a negative one. Buyers with a smaller stock status are thought to have a greater initiative to make their next purchase. The value of this variable is calculated as the difference between the last purchased quantity and the product of the elapsed time and the rate of consumption. 
In addition to these two, there is a third, equally significant, variable $\mathbf{C V}^{\mathbf{h}}{ }_{\text {tr }}$. It talks about the customer's attachment to a specific product. Its primary purpose is to manage changes in probability that arise from the ability of a customer to choose a third-party product.

Some of the problems with this approach are:

- The rate calculated during the initialization period is never adjusted.

- Customer growth and product seasonality are not modeled.

One difference of this approach to the problem domain is the use of the $\mathbf{C V}^{\mathbf{h}}{ }_{\text {tr }}$ parameter. As already pointed out, the purpose of this parameter is to introduce competition into the model. Basically, it is not necessary. Of interest to the problem is any purchase that happens, regardless of the origin of the product. The reason for this is the potential influence on the customer from the perspective of choosing a supplier.

An approach similar to this can be found in [3]Error! Reference source not found.. The authors in this paper combine the choice of time of purchase and brand in one model. The assumption is that the buyer first comes into a situation where it is necessary for him to make a purchase, and then he chooses the manufacturer. Since the purchase moment is of the most interest in this paper, we will focus on that part. The paper defines the frontier of purchases. This limit computes based on two variables:

$$
\begin{gathered}
\mathbf{F R E Q}^{\mathbf{i}}-\text { frequency of purchase } \\
\mathbf{L} \mathbf{Q}_{\mathbf{t}}^{\mathbf{i}}-\text { the normalized amount of the last purchase }
\end{gathered}
$$

Similar as in the previous paper where the customer's consumption rate is calculated during the initialization period and is never changed again, here as well, the purchase frequency is also calculated only during this period and is valid for the entire lifetime of the customer. $\mathbf{L} \mathbf{Q}_{\mathbf{t}}^{\mathbf{i}}$ is used to bring in the effects of buying different quantities of the product. This variable is calculated as the last purchased quantity minus the average purchased quantity in the initialization period.

The purchase frontier is further used in the work as a basis for making purchasing decisions. For each moment, each vendor is assigned a value that indicates the 'desirability' of that vendor. This value depends on the collection of marketing variables which define each vendor at that moment. Transactions occur when the desirability of a vendor exceeds the purchasing frontier.

A more advance modeling was later used in [4] that combines the RFM ([5], [6]) model and the ARIMA model [7].

\subsection{When to buy?}

This group of solutions, as noted above, is characterized by modeling the time between two purchases. The general approach is based on finding a distribution or hazard function that best describes time periods between transactions, parameterizing this distribution / hazard function for each customer-product pair, and then applying it to a specific instance.

The first attempt to find the distribution was seen in [8]Error! Reference source not found.. Here, an exponential distribution was used for modeling purposes. The downside of this approach is that, with exponential distribution, it is by no means possible to take advantage of information that the customer has not yet purchased the product. That is, it is impossible to find the probability of a purchase in the coming period, provided that the 
buyer has not yet purchased the product. One of the earlier attempts to solve this problem can be found in [9]Error! Reference source not found.. This is where authors choose Erlang-2 for distribution function. Although the distribution chosen in this way has produced good results, it cannot be considered universal and of course it cannot be applied to every problem. An overview of the characteristics of distributions found in this field, as well as their specific use cases, is given in [10]Error! Reference source not found.. The distributions covered in this paper are: Exponential, Erlang-2, Weibull, log-logistic and expo-power.

The first model (Pareto/NBD) that has found application in the real world and is still used today was published in [11]. In this model, in addition to the time between two purchases, the customer churn probability is modeled in parallel. The authors in this paper use the Poisson distribution to model the number of purchases a customer makes in a period (the assumption is that the time between two purchases follows an Exponential distribution), while using the Exponential distribution to model the customer lifetime.

Purchase frequency and customer lifetime, at the population level, are modeled using Gamma distributions. In [12] the Markov chain the Monte Carlo model was used to better estimate the parameters of the Pareto/NBD model.

Additional alternatives to this model were later published in [13] (BG/NBD) and [14] (BG/BB). In the former, the Geometric distribution is used instead of the Exponential Distribution to model the customer's lifetime (the assumption being that the customer becomes inactive with a fixed probability after each purchase). At the population level, as with the Pareto/NBD model, the Gamma distribution is used to model the time between purchases while using the Beta-Geometric distribution for the lifetime. For the latter, in addition to the Geometric distribution for lifetime modeling, an additional change is that the Beta distribution is used to model the number of transactions. The idea here is that the buyer has discrete opportunities when he can make a purchase. Similar to the BG / NBD model, at the population level, the Beta-Geometric distribution is used to model customer lifetime, while the Beta distribution is used to model the time between two purchases. Many improvements were later developed for these two models. Some examples of this for the BG/NBD model can be seen in [15] and [16].

Later in [17], another addition was added to the previous models. A gamma/gamma model was added to model the monetary values of customer transactions. Here the assumption is made that the number of transactions a customer makes, and the value of each transaction, are completely independent. An alternative to this was seen in [18], where these two are modeled together.

Another progress on this topic was seen in [19]. Most papers that take the second approach, that is, model the time between two purchases, use the last purchase time as a starting point. In this approach, the same model, calculated during the initialization period, is re-applied each time after a new purchase. The downside to this approach is that the purchase history is never considered when re-calculating. In addition to this problem, if discrete measures of time are expressed in weeks or months, it is not possible to predict two or more purchases in the same period using such models. As a solution, the author suggests using calendar time instead of elapsed time. The goal is to include all available information in the model, from the first purchase up until the moment of calculations. Another benefit of using calendar time is the ability to add seasonality. To achieve this, four components are used: calendar elapsed time, elapsed time between 
purchases, covariance functions, and deviation from the mean time between purchases. In addition to these components, the author also adds 'at-risk' variables in his model to determine the likelihood of successive purchases as well as purchases on specific days. The application of these variables, in addition to controlling multiple purchases at a time, is, for example, assigning probabilities to purchases at certain moments. In the wholesale world, for example, it might be important to set the probability of customers buying any products on Saturdays and Sundays to zero.

Regardless of the choice of approach, it is important to note that no solution is universal and, to a large extent, depends on the specific use case. This results in the fact that when applying one of the solutions to a different data set, it is necessary to allocate additional time to investigate the specific case of application as well as to parameterize the models used.

\section{IMPLEMENTED SOLUTIONS}

For comparison purposes, four different algorithms have been implemented and used to predict future moments of purchases. The ARIMA model was used as a representative of the first group of solutions mentioned in the previous chapter (0). From the second group (0) the Pareto/NBD model and its modifications in the form of a Poisson distribution were implemented. For the sake of comparison, the Naive forecasting was also added as a base of comparison. Each one will be discussed below.

\section{A. ARIMA}

As noted above, given that the data being analyzed does not represent a normal time series, it must first be properly transformed. It is for this reason that the first step in the process of analyzing and predicting future values is to prepare data.

After loading all purchases made by a particular consumer, the average daily consumption of a product is calculated up to the desired date. Going through a series of purchases, for each two purchases, the number of days between them is calculated, and then dividing the purchased quantity in the first by the number of days gives an average daily consumption for the period between those two specific purchases. In this way, a regular time series of daily product consumption is obtained from the initial set of discrete purchases. This process must be performed for each product that the customer purchases, and additionally, for each consumer in the system. In addition, a series of average daily consumption is loaded directly from the database, but at the product level, i.e. daily product consumption by all customers in the system. The reason for using this extra set is a more accurate consumption forecast for customers with a scarce shopping history.

After obtaining a regular time series, one can start analyzing and predicting future values using the ARIMA model. All calculations at this stage related to time series modeling were performed in the R software language due to the availability of adequate packages.

Based on the last recorded purchase quantity of a particular product, it is with certain probability that the consumer will consume all supplies of that product within the next week. If this is the case, then that consumer and item pair is added to the list of expected purchases if its consumption is within the limits of what is considered reasonable. 


\section{B. Pareto/NBD}

Unlike the ARIMA model, the Pareto/NBD model's input data do not need to first be transformed. This model requires only the purchase frequency and the elapsed time since the last purchase. However, in order to use the information on the quantity of products purchased, the total purchased quantity is used instead of the number of transactions for the frequency calculation. That is, the unit consumption rate of a product is calculated, and this is used instead of the purchase frequency.

After this, as with the ARIMA model, all calculations related to time series modeling were performed in $\mathrm{R}$ software language. Using the forecast package, the estimated number of purchases by the end of next week is calculated (i.e. since instead of the frequency of purchases, the unit consumption rate of the product was fed into the model, the output is going to be the expected consumption of the product by the end of the week). If this value is greater than the quantity of the product purchased at the last purchase, it is considered that the buyer will make another purchase in the forthcoming period.

In theory, the Pareto/NBD model can be created once for the entire population level, but this was not possible in this case due to limitations in the form of memory resources needed, so the processing is divided into several stages. In each step, processing is performed for all of the products for one customer. Thus, all of the products for each customer are considered to be independent, that is, the assumption is that the consumptions of each product are independent.

\section{Poisson distribution}

As stated earlier this is just a modification of the Pareto/NBD model. The first difference is that in this approach, the necessary modeling is reduced to a minimum. The Pareto/NBD model searches for the parameters of Gamma distributions at the population level, which is useful if we are interested in the general characteristics, such as the movement of the global trend in the number of purchases, the average customer lifetime, etc. However, for the very domain of this problem (predicting the next purchases), it is not helpful. As this is not very useful and has an impact on performance, this part has been dropped.

Another change concerns the way in which the probability of a customer churn is predicted. In the Pareto/NBD model, as mentioned above, the customer lifetime is modeled by Exponential Distribution. Instead, the Poisson's distribution is again used as a part of this approach. For the purpose of calculating the probability of a purchase, two probabilities are calculated.

First, the probability that the buyer will run out of stock between the last purchase date and the current date. This probability is considered to be directly proportional to the probability of the customer churning (the greater the likelihood that the customer consumes all of the supplies during this period, the greater the likelihood that they abandoned this product). In addition, the probability that the buyer will run out of supplies by the end of the week is also calculated in a similar way as with the Pareto/NBD model. This value is directly proportional to the probability of a customer making a purchase. 


\section{Naive forecasting}

For comparison purposes, a version of the naive forecasting method was implemented. This method finds the moment of the next purchase by calculating three parameters for each customer-product pair: the mean, minimum and maximum time between two purchases. Using these parameters, the time of the next purchase was obtained by adding the mean time between two purchases at the time of the last purchase, provided that there are no major deviations from the minimum and maximum time. The allowed difference between the two parameters is obtained by striking a balance between the precision of the prediction and the number of predictions to be made. If a large difference were used, precision would drop dramatically. If that difference were too small, then there would not be enough predictions for the next week.

\section{4, METHOD COMPARISON}

All methods were tested against real data from medical device manufacturers.

The data we worked with consist of over seven and a half million sales transactions collected over four years and five months. The set is made up of just over 12,000 unique customers, of whom about 10,200 have had repeat purchases. On the other hand, about 10,600 unique products were sold during this period. For testing purposes, predictions were made on Sundays for a period of next week. The average number of transactions on a weekly basis during the test period was approximately 31,500 . The shopping period was divided into:

- Training and validation set $-50 \%$

- Test set - 50\%

The reason for this split came from the sub-section for an unusually large test set. Namely, since all the purchase predictions, not just the ones related to the supplier whose data we use, are expected to exit the system, it was necessary to remove the buyers and the purchase predictions that never happened again. In such cases, we assume that the purchases were made with another supplier or that the buyer found another alternative to the product. As we cannot be sure when a purchase in this group occurred at another vendor, we must remove it from the statistics. To ensure that the purchase did not occur, the test period must be large enough to remove any doubt.

All models were compared by four criteria:

- the percentage of purchases made relative to the number of predicted purchases (True predictions)

- the percentage of purchases made relative to the number of predicted purchases excluding products that were never purchased again (True predictions excluding abandoned items)

- percentage of projected purchases compared to the number of realized purchases (Purchase coverage)

- the percentage of predicted purchases over the number of realized purchases, taking only those who have had at least two previous purchases (Purchase coverage (at least two prior purchases)

The results of the comparison are given in the Fig. 1. 


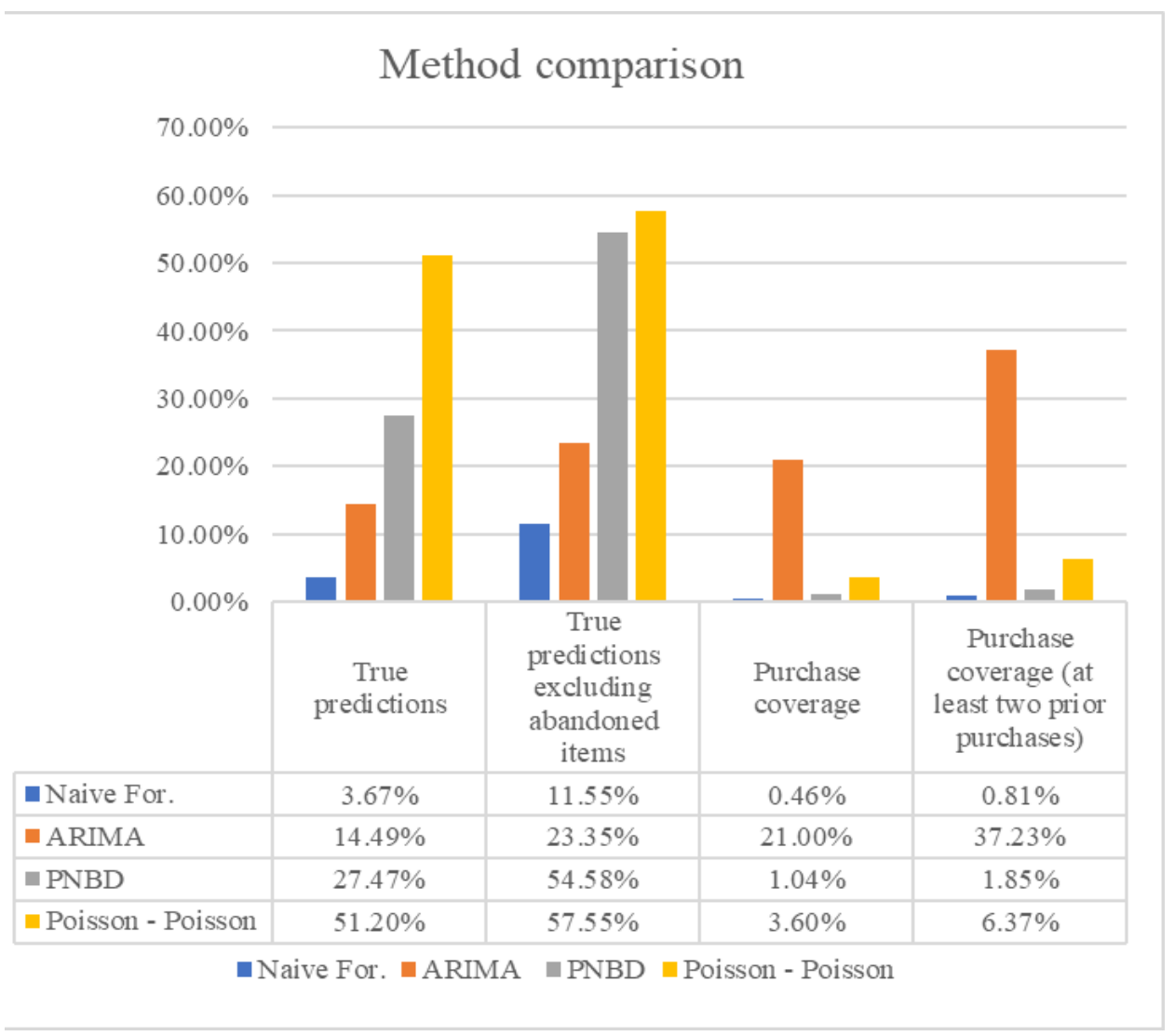

Fig. 1 Method comparison

It can be seen immediately that all three models deliver dramatically better results in all categories than naive prediction. In addition, it can be seen that the Pareto / NBD and Poisson distribution are more accurate while ARIMA covers a larger number of cases. This can be attributed to the fact that ARIMA does not in any way predict the dying away of customers, that is, the extinction of extinct customers is done in a very primitive way (only expenditures that exceed the amount previously purchased are eliminated). It is also interesting that the Poisson distribution with modified identification of extinct customers gave better results than the Pareto / NBD model. This can be overwritten by the specifics of the problem with the characteristics of the B2B business model (general stability of the purchasing process).

An interesting comparison of these models can also be made on the basis of their performance, that is, the execution time (given in Fig. 2). 


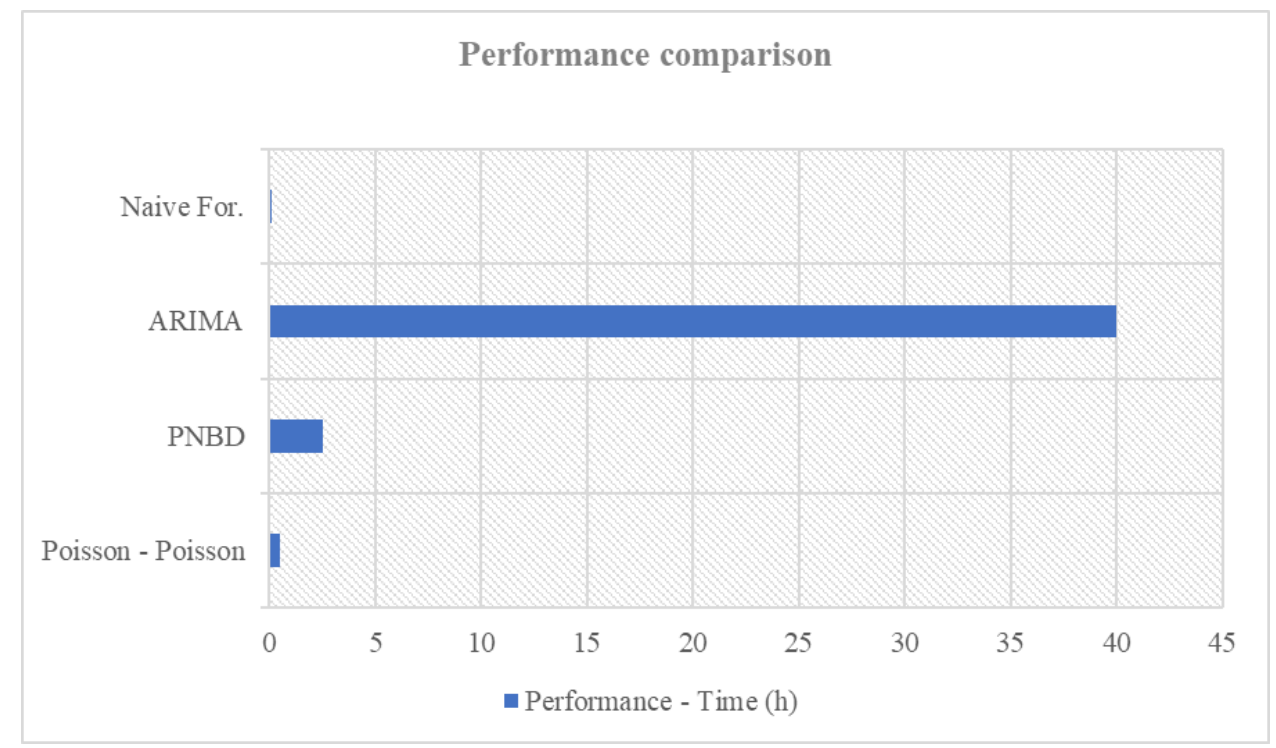

Fig. 2 Performance comparison

In terms of performance, the worst results were given by ARIMA, the reason being that a complex model is created at the customer-product level, and communication with $\mathrm{R}$ is very demanding. The reason Poisson's distribution had better performance than the Pareto / NBD distribution is because the model being created is more primitive, that is, there are no population-level parameters.

All data processing was done on a computer with an Intel brand i7-6700T processor and $8 \mathrm{~GB}$ of RAM. The complexity of the algorithm is linearly dependent on the number of customer-product pairs, and this has favorable scalability outcomes. Also, the solutions can be largely parallelized and have elements of computation that can potentially be executed on the GPU, which would further increase performance.

\section{CONCLUSION}

Time series models are a very powerful tool for predicting future values and making decisions in different areas of life and work: economy, agriculture, industry, medicine, etc. It has been shown in practice that a large number of time-dependent phenomena can be modeled by stochastic processes. The prevalence and attractiveness of these models is due to their very structure, which is easy to understand and quite intuitive.

Of course, like all models, the ones used in this paper are not a perfect representation of the real state of affairs, but nevertheless, they still allow for a fast and efficient procedure that gives a lot of precision with the forecast of future values. Depending on the dataset, as well as on the requirements, different models should be tried out and tested against the specific dataset. As it was shown in previous chapter ([0]), the ARIMA model covers a larger number of data, while the Pareto / NBD and Poisson distribution are more accurate. 
Depending on the purpose of the work, and the required level of performance, the appropriate forecasting model should be chosen.

Besides the models described in this paper, there are many others that depending on the nature of data, may be better fit for forecasting. Many forecasting methods depend on the use of neural networks. Introduction in using neural networks for forecasting of financial markets may be found in [20]. Methods introduced here provide the necessary basis in further research work on this topic. More advanced neural networks are described in [21]. Here, authors start from implementing simple neural network and comparing its forecasting results against traditional Black-Scholes model. However, by far the best results are given by the hybrid model between the first two.

Since neural networks are very common in time-series analysis and have also been proven to provide satisfying results in forecasting future values, they will certainly be the focus of future work on this topic.

\section{REFERENCES}

[1] http://www.ijstr.org/final-print/sep2014/A-Comparative-Study-On-B2b-Vs-B2c-Based-On-Asia-PacificRegion.pdf.

[2] Bucklin, Randolph E., and Sunil Gupta. 1992. "Brand Choice, Purchase Incidence, and Segmentation: An Integrated Modeling Approach.” Journal of Marketing Research 29 (2): 201-215.

[3] Zhang, Jie, and Lakshman Krishnamurthi. 2004. "Customizing Promotions in Online Stores." Marketing Science 23 (4): 561-578

[4] Khajvand, M., \& Tarokh, M. J. (2011). Estimating customer future value of different customer segments based on adapted RFM model in retail banking context. Procedia Computer Science, 3, 1327-1332. doi:10.1016/j.procs.2011.01.011.

[5] Wei, Jo-Ting \& Lin, Shih-Yen \& Wu, Hsin-Hung. (2010). A review of the application of RFM model. African Journal of Business Management December Special Review. 4. 4199-4206.

[6] Dogan, Onur \& Ayçin, Ejder \& Bulut, Zeki. (2018). CUSTOMER SEGMENTATION BY USING RFM MODEL AND CLUSTERING METHODS: A CASE STUDY IN RETAIL INDUSTRY. International Journal of Contemporary Economics and Administrative Sciences. 8. 1-19.

[7] Box, G. and Jenkins, G. (1970) Time Series Analysis: Forecasting and Control. Holden-Day, San Francisco.

[8] Ehrenberg, A.S.C. 1959. "The Pattern of Consumer Purchases." Applied Statistics 8: 26-41.

[9] Gupta, Sunil. 1988. "Impact of Sales Promotions on When, What, and How Much to Buy." Journal of Marketing Research 25 (4): 342-355.

[10] Seetharaman, P. \& Chintagunta, Pradeep. (2003). The Proportional Hazard Model for Purchase Timing: A Comparison of Alternative Specifications. Journal of Business \& Economic Statistics. 21. 368-82. 10.1198/073500103288619025.

[11] Fader, Peter \& Hardie, Bruce \& Lee, Ka. (2005). "Counting Your Customers" the Easy Way: An Alternative to the Pareto/NBD Model. Marketing Science. 24. 275-284. 10.1287/mksc.1040.0098.

[12] Ma, Shao-Hui \& Liu, Jin-Lan. (2007). The MCMC approach for solving the Pareto/NBD model and possible extensions. Proceedings - Third International Conference on Natural Computation, ICNC 2007. 2. 505 - 512. 10.1109/ICNC.2007.728

[13] Fader, Peter \& Hardie, Bruce \& Lee, Ka. (2005). "Counting Your Customers" the Easy Way: An Alternative to the Pareto/NBD Model. Marketing Science. 24. 275-284. 10.1287/mksc.1040.0098.

[14] Fader, Peter \& Hardie, Bruce \& Huang, Chun-Yao. (2004). A Dynamic Changepoint Model for New Product Sales Forecasting. Marketing Science. 23. 50-65. 10.1287/mksc.1030.0046.

[15] van Oest, Rutger \& Knox, George. (2010). Extending the BG/NBD: A Simple Model of Purchases and Complaints. International Journal of Research in Marketing. 28. 10.1016/j.ijresmar.2010.11.001.

[16] Ben Mzoughia, Mohamed \& Limam, Mohamed. (2014). An Improved BG/NBD Approach for Modeling Purchasing Behavior Using COM-Poisson Distribution. International Journal of Modeling and Optimization. 4. 10.7763/IJMO.2014.V4.362

[17] Fader, P. S., Hardie, B. G. S. and Lee, K. L. (2005) 'RFM and CLV: Using Iso-Value Curves for Customer Base Analysis', Journal of Marketing Research, 42(4), pp. 415-430. doi: 10.1509/jmkr.2005.42.4.415. 
[18] Glady, Nicolas \& Baesens, Bart \& Croux, Christophe. (2007). A modified Pareto/NBD approach for predicting customer lifetime value. Katholieke Universiteit Leuven, Open Access publications from Katholieke Universiteit Leuven. 36. 10.1016/j.eswa.2007.12.049.

[19] Bijwaard, Govert E. 2006. "Regularity in Individual Shopping Trips: Implications for Duration Models in Marketing.” Working paper, Econometric Institute, Erasmus University, Rotterdam.

[20] E. Michael Azoff (1994) "Neural Network Time Series Forecasting of Financial Markets".

[21] Hosein Marzi, Mark Turnbull (2007). "Use of Neural Networks in Forecasting Financial Market". 2007 IEEE International Conference on Granular Computing (GRC 2007). 